\title{
DIRECT DEBs in M31 and M33
}

\author{
Lucas M. Macri \\ Hubble Fellow, National Optical Astronomy Observatory, $950 \mathrm{~N}$ Cherry \\ Avenue, Tucson, AZ 85719, USA
}

The DIRECT project (as in "direct distances") started in 1996 with the longterm goal of obtaining distances to two important galaxies in the cosmological distance ladder - M31 and M33 - using detached eclipsing binaries (DEBs) and Cepheids. These two nearby galaxies are the stepping stones in most of the current effort to understand the evolving universe at large scales. Not only are they essential to the calibration of the extragalactic distance scale, but they also constrain population synthesis models for early galaxy formation and evolution. However, accurate distances are essential to make these calibrations free from large systematic uncertainties.

Detached eclipsing binaries have the potential to establish distances to M31 and M33 with an unprecedented accuracy of better than $5 \%$ and possibly to better than 1\%. Current uncertainties in the distances to these galaxies are in the order of 10 to $15 \%$, as there are discrepancies of $0.2-0.3$ mag between various distance indicators. Detached eclipsing binaries (Paczyński 1997) offer a single-step distance determination to nearby galaxies (Fitzpatrick et al. 2003) and may therefore provide an accurate zero-point calibration for other distance indicators, including Cepheids.

The DIRECT project obtained time-series observations of M31 and M33 during 170 nights between 1996 September 6 and 2000 January 2 using the Fred L. Whipple Observatory 1.2-m telescope. Additional observations were carried out during 36 nights in 1996 and 1997 at the Michigan-Dartmouth-MIT Observatory 1.3-m telescope. In 1996 and 1997, images were obtained using a camera with $\sim 11^{\prime} \times 11^{\prime}$ field of view. In 1998 and 1999, data were collected using a camera with $a \sim 22^{\prime} \times 22^{\prime}$ field of view. Observations were obtained mainly in the $V$ and $I$ bands, with some additional data in the $B$ band. The total area covered by the observations was $\sim 0.5 \square^{\circ}$ in M31 and $\sim 0.3 \square^{\circ}$ in M33.

Most of the fields have been analyzed and published (Bonanos et al. 2003; Kaluzny et al. 1998; Kaluzny et al. 1999; Macri et al. 2001c; Mochejska et al. 1999; Stanek et al. 1998; Stanek et al. 1999). These publications contain a total of $\sim 130$ eclipsing binaries, $\sim 600$ Cepheid variables, and $\sim 500$ miscellaneous variables.

Additional publications related to the DIRECT project include: the discovery of cessation of pulsations in a long-period Cepheid in M33 (Macri et al. 2001a); the study of the influence of unresolved blends on the Cepheid Distance Scale (Mochejska et al. 2000; Mochejska et al. 2001a); a catalog of globular clusters in M31 and M33 (Mochejska et al. 1998); ancillary stellar catalogs and additional short-period variables (Macri et al. 2001b; Mochejska et al. 2001b; Mochejska et al. 2001c; Mochejska et al. 2001d). 
Four promising detached eclipsing binary systems have been discovered in the DIRECT fields; two in M31 and two in M33. As a first step in their followup, higher quality light curves were obtained through observations conducted at the Kitt Peak National Observatory 2.1-m telescope in 1999 and 2001. Recently, a program was started to measure radial velocities of the DEBs using the Echelle Spectrograph and Imager (ESI) at Keck Observatory. We have also obtained out-of-eclipse near-infrared observations of the DEBs in M33 using the Gemini North 8-m telescope, to increase the wavelength baseline of our coverage and obtain a better measurement of $E(B-V)$ for these systems.

\section{References}

Bonanos, A. Z., Stanek, K. Z., Sasselov, D. D., Mochejska, B. J., Macri, L. M., \& Kaluzny, J. 2003, AJ, 126, 175

Fitzpatrick, E. L., Ribas, I., Guinan, E. F., Maloney, F. P., \& Claret, A. 2003, ApJ, 587,685

Kaluzny, J., Stanek, K. Z., Krockenberger, M., Sasselov, D. D., Tonry, J. L., \& Mateo, M. 1998, AJ, 115,1016

Kaluzny, J., Mochejska, B. J., Stanek, K. Z., Krockenberger, M., Sasselov, D. D., Tonry, J. L., \& Mateo, M. 1999, AJ, 188, 346

Macri, L. M, Sasselov, D. D. \& Stanek, K. Z. 2001a, ApJ, 550, L159

Macri, L. M., Stanek, K. Z., Sasselov, D. D., Krockenberger, M., \& Kaluzny, J. 2001b, AJ, 120, 861

Macri, L. M., Stanek, K. Z., Sasselov, D. D., Krockenberger, M., \& Kaluzny, J. 2001c, AJ, 120,870

Mochejska, B. J., Kaluzny, J., Krockenberger, M., Sasselov, D. D., \& Stanek, K. Z. 1998, AcA, 48, 445

Mochejska, B. J., Kaluzny, J., Stanek, K. Z., Krockenberger, M., \& Sasselov, D. D. 1999, AJ, 118, 2211

Mochejska, B. J., Macri, L. M., Sasselov, D. D., \& Stanek, K. Z. 2000, AJ, 120, 810

Mochejska, B. J., Macri, L. M., Sasselov, D. D., \& Stanek, K. Z. 2001a, astro-ph/0103440

Mochejska, B. J., Kaluzny, J., Stanek, K. Z., \& Sasselov, D. D. 2001b, AJ, 122, 1383

Mochejska, B. J., Kaluzny, J., Stanek, K. Z., Sasselov, D. D., \& Szentgyorgyi, A. H. 2001c, AJ, 121, 2032

Mochejska, B. J., Kaluzny, J., Stanek, K. Z., Sasselov, D. D., \& Szentgyorgyi, A. H. $2001 \mathrm{~d}, \mathrm{AJ}, 122,2477$

Paczyński, B. 1997, in The Extragalactic Distance Scale, ed. M. Livio, M. Donahue \& N. Panagia (Cambridge: Cambridge Univ. Press), 273

Stanek, K. Z., Kaluzny, J., Krockenberger, M., Sasselov, D. D., Tonry, J. L., \& Mateo, M. $1998, \mathrm{AJ}, 115,1894$

Stanek, K. Z., Kaluzny, J., Krockenberger, M., Sasselov, D. D., Tonry, J. L., \& Mateo, M. $1999, \mathrm{AJ}, 117,2810$ 